\title{
Complexity Management Using Metrics for Trajectory Flexibility Preservation and Constraint Minimization
}

\author{
Husni R. Idris ${ }^{*}$ and Ni Shen ${ }^{\dagger}$ \\ Engility Corporation, Billerica, MA, 01821 \\ David J. Wing ${ }^{\ddagger}$ \\ NASA Langley Research Center, Hampton, VA, 23681
}

\begin{abstract}
The growing demand for air travel is increasing the need for mitigating air traffic congestion and complexity problems, which are already at high levels. At the same time new surveillance, navigation, and communication technologies are enabling major transformations in the air traffic management system, including net-based information sharing and collaboration, performance-based access to airspace resources, and trajectorybased rather than clearance-based operations. The new system will feature different schemes for allocating tasks and responsibilities between the ground and airborne agents and between the human and automation, with potential capacity and cost benefits. Therefore, complexity management requires new metrics and methods that can support these new schemes. This paper presents metrics and methods for preserving trajectory flexibility that have been proposed to support a trajectory-based approach for complexity management by airborne or ground-based systems. It presents extensions to these metrics as well as to the initial research conducted to investigate the hypothesis that using these metrics to guide user and service provider actions will naturally mitigate traffic complexity. The analysis showed promising results in that: (1) Trajectory flexibility preservation mitigated traffic complexity as indicated by inducing self-organization in the traffic patterns and lowering traffic complexity indicators such as dynamic density and traffic entropy. (2)Trajectory flexibility preservation reduced the potential for secondary conflicts in separation assurance. (3) Trajectory flexibility metrics showed potential application to support user and service provider negotiations for minimizing the constraints imposed on trajectories without jeopardizing their objectives.
\end{abstract}

$\begin{array}{lll}\mathrm{ADP} & = & \text { Adaptability } \\ \mathrm{RBT} & = & \text { Robustness } \\ \mathrm{RTA} & = & \text { Required Time of Arrival } \\ \mathrm{k},(\mathrm{t}, \mathrm{s}) \text { or }(\mathrm{t}, \mathrm{x}, \mathrm{y}, \mathrm{z}) & = & \text { Point in space and time also denoted as (time, x-location, y-location, z-location) } \\ \mathrm{Traj} & = & \text { Projectory } \\ \mathrm{P}_{\mathrm{f}} & = & \text { Number of feasible trajectories from } \mathrm{k} \text { to destination } \\ \mathrm{f}(\mathrm{k}) & = & \text { Number of infeasible trajectories from } \mathrm{k} \text { to destination } \\ \mathrm{i}(\mathrm{k}) & = & \text { Number of all trajectories from k to destination } \\ \mathrm{N}(\mathrm{k}) & = & \text { Number of trajectories from } \mathrm{k} \text { to next time step } \\ \mathrm{g}(\mathrm{k}) & & \text { Duration between time increments } \\ \varepsilon & \end{array}$




\section{Introduction}

$\mathrm{T}$ HE Next Generation Air Transportation System (NextGen) is expected to receive two to three times the current traffic demand by the year 2025. ${ }^{1}$ In order to handle this increase in air traffic, NextGen will introduce key transformations in Air Traffic Management (ATM). Three examples of the transformations are: increasing information sharing through net-enabled information access; making access to National Airspace System (NAS) resources dependent on aircraft equipage; and aircraft trajectory-based operations enabled by aircraft ability to precisely follow customized four dimensional (4D) trajectories. ${ }^{1}$ These transformations enable different schemes for allocating tasks between ground-based and airborne agents and between the human and the automation, with potential capacity and cost benefits. For example, NextGen is investigating delegating more responsibility for traffic separation to the pilot and delegating more responsibility to flight operation centers for traffic flow management. ${ }^{2,3,4}$

This paper summarizes research that has been conducted to investigate two newly proposed functions for the ATM system: trajectory flexibility preservation and trajectory constraint minimization. ${ }^{5,6}$ The two functions offer a trajectory-oriented approach to managing traffic complexity by explicitly planning aircraft trajectories such that their contribution to complexity is minimized. This is contrasted with airspace-oriented approaches that aim to ensure that airspace structure (such as sector size and route patterns) and traffic characteristics (such as aircraft density) are planned to dynamically limit traffic complexity. Trajectory flexibility preservation enables planning an aircraft's trajectory such that it preserves a requisite level of maneuvering flexibility in accommodating disturbances, caused for example by other traffic or by weather activity. Trajectory constraint minimization enables ground-based agents, in collaboration with air-based agents, to impose just-enough constraints on trajectories to achieve ATM objectives, such as separation assurance and flow management. The concept hypothesizes that by preserving trajectory flexibility, aggregate system objectives, such as maintaining acceptable traffic complexity, are naturally achieved, and that minimizing the constraints imposed on a trajectory, without jeopardizing the intended ATM objectives, increases its flexibility.

Trajectory flexibility preservation complements separation assurance both within the conflict resolution horizon and beyond it to an extended flexibility planning horizon. Beyond the conflict resolution horizon, flexibility preservation plans the aircraft trajectory to minimize its exposure to disturbances such as weather cells and dense traffic. Figure 1 depicts an example. In its left side each aircraft, while planning its trajectory between weather cells, questions whether it should modify its trajectory to increase flexibility. If the aircraft proceed along their depicted headings, a complex traffic situation arises causing excessive congestion and a high potential conflict rate. On the other hand, the right side displays a structured traffic pattern that would result if each aircraft maneuvered to increase its own flexibility.

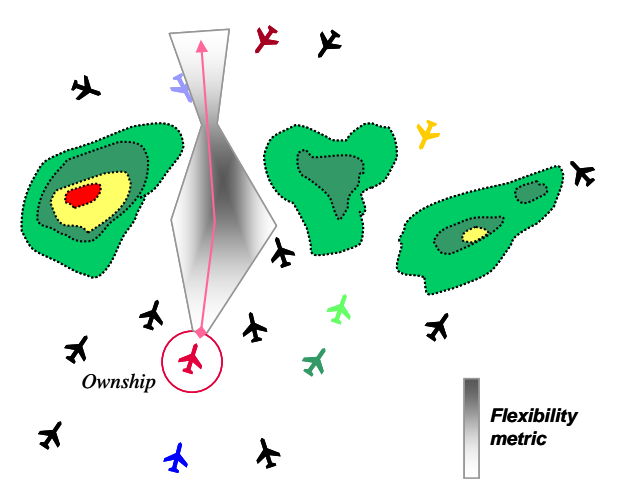

Airborne flexibility function will question:

Do I have enough flexibility to safely proceed? Can I modify my trajectory to increase my flexibility? Do I need to avoid this airspace entirely and replan?
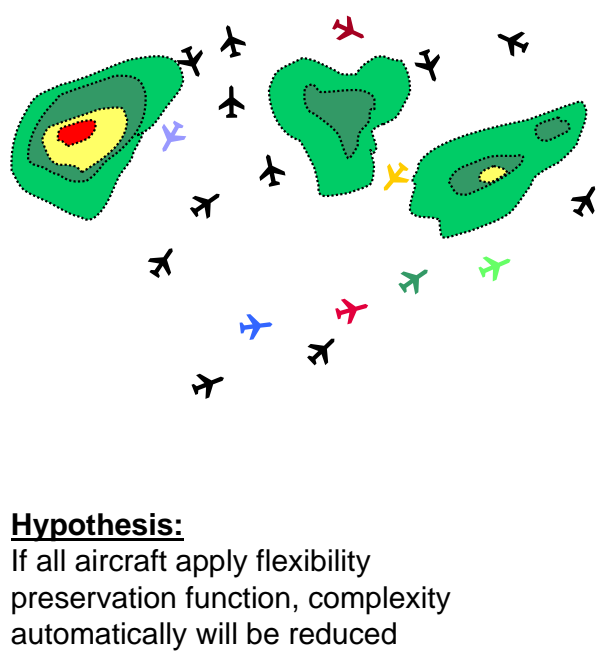

Figure 1. Complexity mitigation through trajectory flexibility preservation

Within the conflict resolution horizon, flexibility is used to select from many conflict resolution solutions one that affords the aircraft more flexibility, for example to adapt to potential intruder traffic behavior. One example is 
shown in Figure 2, where in the left side two conflicts are predicted within the look-ahead horizon between two unrelated pairs of aircraft. If the two aircraft labeled "ownship" maneuvered to resolve their respective predicted conflict, without coordination, a new coincidental conflict may arise between them as shown by the dashed lines. Although flexibility preservation does not explicitly coordinate between the two ownship aircraft, it assists each one in reducing the risk of conflict due to the potential behavior of the surrounding traffic, thus resulting in implicit coordination. Hence with this function, each ownship aircraft may select a more flexible trajectory anticipating the potential maneuver of the other aircraft and maneuvering away from it as shown in the right side of Figure 2.
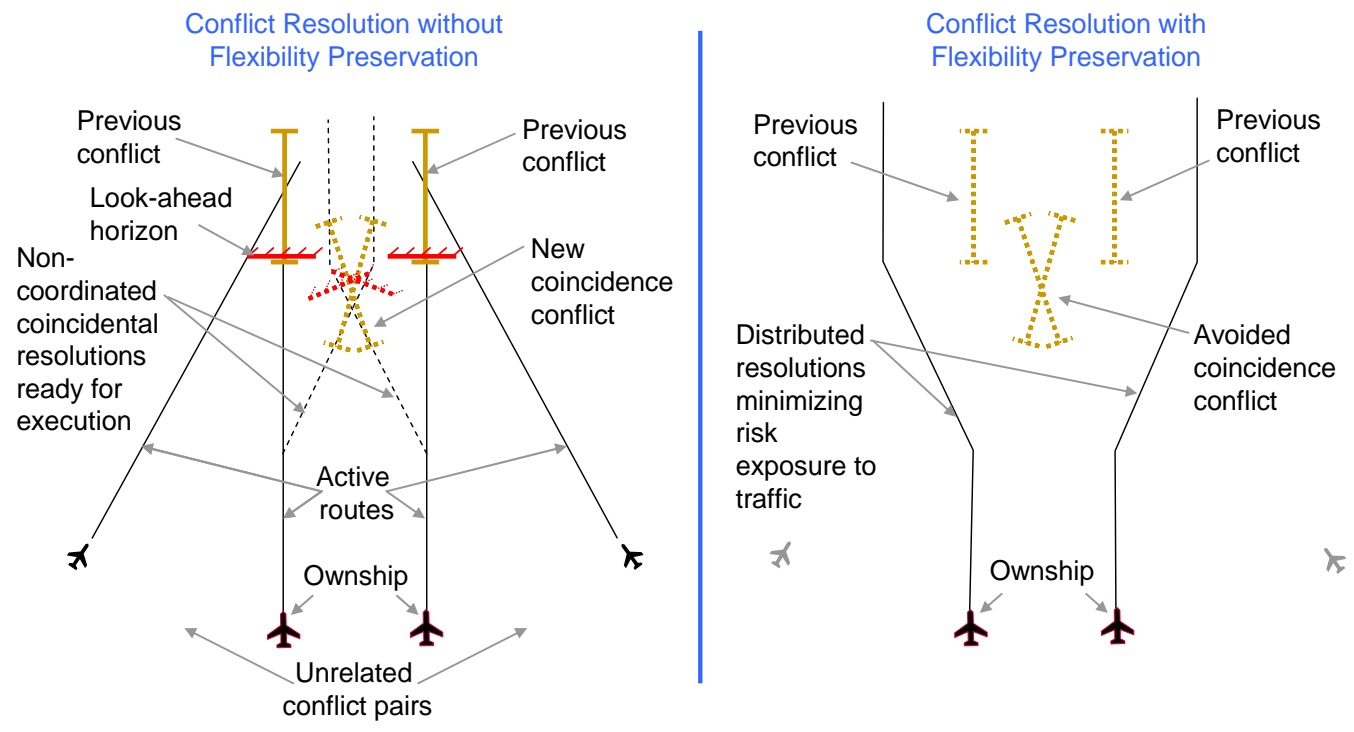

Figure 2. Flexibility preservation avoiding coincidence conflict

Trajectory constraint minimization assists a controller or traffic manager, in collaboration with the pilot or flight operator, to impose minimal constraints on the aircraft to meet an intended ATM objective. For example, if a single required time of arrival (RTA) at a specified fix will sufficiently meter the traffic flow, multiple RTAs per aircraft are deemed too excessive and hence candidate for relaxation. Trajectory constraint minimization enables more efficient utilization of airspace resources and increases the pilot's ability to maneuver freely with fewer constraints in order to accommodate disturbances. Therefore, the pilot may negotiate constraint reduction from the cockpit perspective. For example, airborne automation may determine that the aircraft cannot meet all its constraints with sufficient flexibility, and hence the pilot may provide information to help the traffic manager relax some constraints. Figure 3 demonstrates the hypothesized impact of constraint minimization on trajectory flexibility preservation and hence traffic complexity. Ownship aircraft 'A' plans its trajectory to resolve a predicted conflict with aircraft 'B' within the conflict resolution look-ahead and to meet an RTA at a downstream fix within the flexibility planning horizon. The RTA tolerance initially allows aircraft 'A' to avoid the predicted conflict only by path stretching to the left, with expected times of arrival (ETA) at the fix that lie within the RTA tolerance (left side of figure). These trajectories expose aircraft ' $\mathrm{A}$ ' to disturbances from nearby traffic (Aircraft $\mathrm{C}$ and D) and an inclement weather system, and they would increase its contribution to traffic complexity. With this information, the traffic manager relaxes the RTA constraint by increasing its tolerance as shown in the right side of the figure, having determined that the RTA's intended ATM objectives can still be met sufficiently. With the extended tolerance, aircraft 'A' has more maneuvering options to avoid the predicted conflict to the right and select a more flexible trajectory with less exposure to the weather and traffic, thus reducing the aircraft contribution to traffic complexity. The aircraft is also able to meet its RTA constraint more accurately with less risk exposure and hence achieve the intended ATM objectives more reliably.

To test the hypothesized impacts of trajectory flexibility preservation and constraint minimization, trajectory flexibility metrics have been defined in previous work to represent robustness and adaptability to the risk of violating separation, airspace hazards, and traffic flow management constraints. ${ }^{5-8}$ In these previous efforts, the authors showed that using trajectory flexibility in a long time horizon mitigated traffic complexity by inducing self 

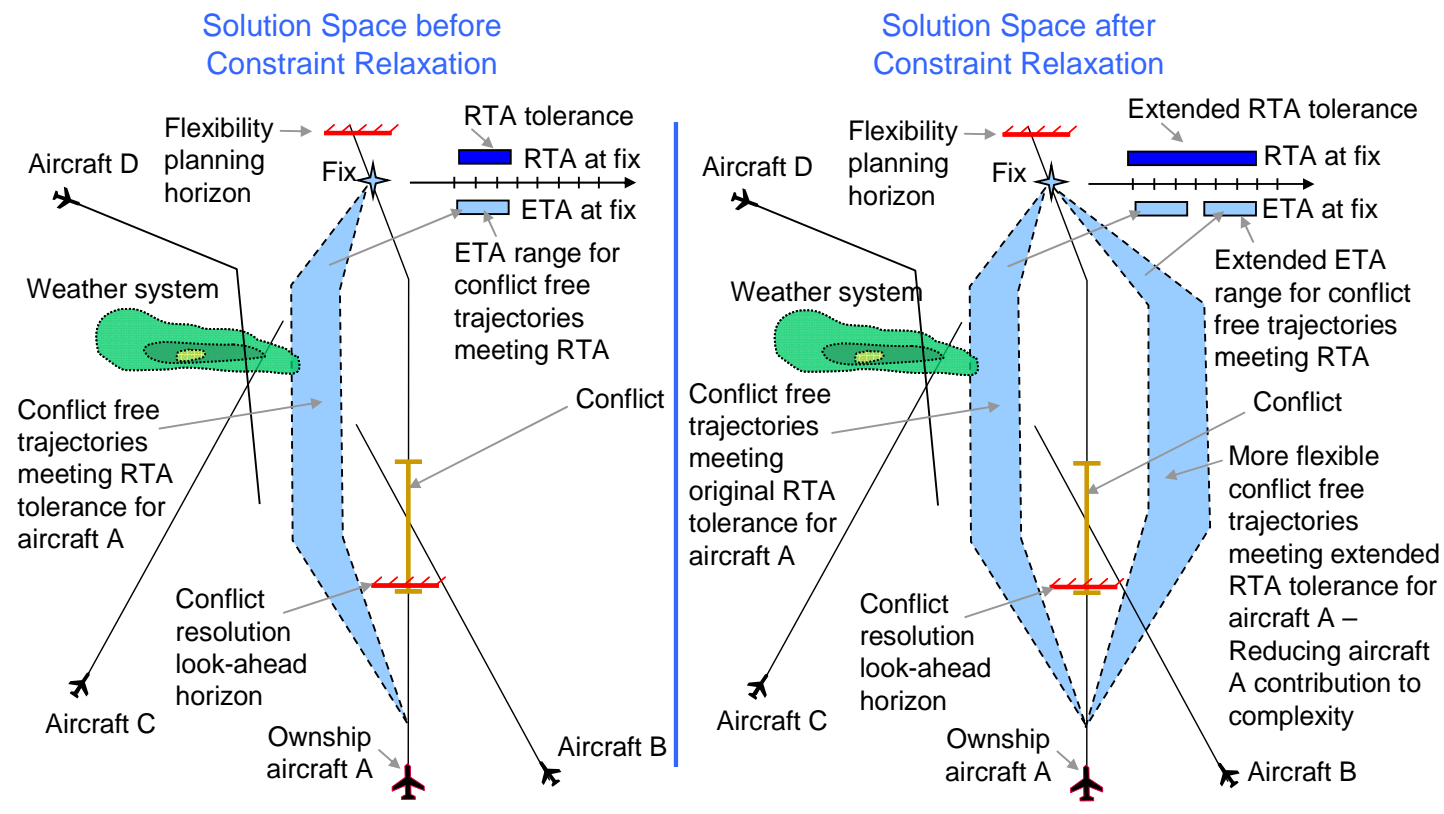

Figure 3. Trajectory constraint minimization - relaxing RTA tolerance

organization in the traffic situation as well as reducing measures of traffic complexity such as dynamic density. ${ }^{9,10}$ The authors also showed in a previous effort that the application of trajectory flexibility metrics reduced the creation of secondary conflicts by bringing about implicit coordination between aircraft. ${ }^{11}$ This paper summarizes the previous results and presents new extensions to the trajectory flexibility metrics and their application in traffic complexity mitigation and constraint minimization. Namely: (1) Additional metrics and variations on the previously suggested metrics are presented. (2) Trajectory flexibility preservation is demonstrated in a three dimensional scenario where it resulted in distributing the aircraft evenly over the allowed altitudes thus reducing congestion in each altitude. (3) Trajectory flexibility metrics are shown to be effective in assessing the amount of maneuverability afforded to the aircraft by the constraints imposed on it and hence its exposure to the risk of constraint violation. Therefore, these metrics may be used to aid negotiating constraint relaxation by the flight crew and minimizing the amount of constraints imposed on the aircraft by the ground based traffic management system.

The paper is organized as follows: First the extensions to the trajectory flexibility metrics are described. Then each area: (1) traffic complexity management, and (2) negotiation of constraint relaxation, is described with analytical examples demonstrating the concept and hypothesis in each.

\section{Trajectory Flexibility Metrics}

Metrics have been proposed to represent the exposure of the aircraft trajectory to the risk of violating constraints imposed on it, and hence to be used to mitigate this risk ${ }^{5-8}$ Constraints are imposed on aircraft trajectories to meet certain objectives, such as imposing an RTA which is intended to help balance demand and capacity of airspace resources. Constraints are also imposed on trajectories in order to avoid safety hazards, such as violation of separation requirements from traffic or weather cells. The violation of the constraints may be caused by uncertainties in the constraints, which were called constraint disturbances; they represent variation in predicted constraints or appearance of new constraints that were unforeseen at the time of trajectory planning. The violation of constraints may also be caused by uncertainties in the aircraft planned states, which were called state disturbances; they represent predicted or unforeseen deviations in the state due to wind or pilot error for example. The following subsections are a summary and extensions of the metrics and estimation methods that have been proposed to measure trajectory flexibility. 


\section{A. Metric definitions}

Since the flexibility of the aircraft trajectory was defined as its ability to mitigate the risk of constraint violation the following characteristics were identified and defined to help derive metrics for measuring this risk and hence flexibility.

1. Robustness (RBT) was defined as the ability of the aircraft to maintain feasibility (i.e., not violating any constraints) despite the occurrence of constraint and state disturbances that pose the risk of constraint violation. Robustness, denoted as RBT, is measured with the probability of feasibility RBT(.) $=\mathrm{P}_{\mathrm{f}}($.$) , where the dot refers$ to the object of which robustness is measured. Hence, the robustness of a specific aircraft trajectory is defined as the probability that the trajectory remains feasible. For example if the trajectory (traj) is defined as a series of points $(\mathrm{k})$ in space and time, and each point has a probability of feasibility $\mathrm{p}_{\mathrm{f}}(\mathrm{k})$ that is independent of all other points, then the probability of feasibility of the trajectory is the multiplication of the independent probabilities of feasibility of all the trajectory points: $\mathrm{RBT}($ traj $\left.)=\mathrm{P}_{\mathrm{f}}(\operatorname{traj})=\Pi_{\mathrm{k}}\left(\mathrm{p}_{\mathrm{f}}(\mathrm{k})\right)\right)$. The robustness of the trajectory solution space, that consists of a set of possible trajectories available to the aircraft at a point $(\mathrm{k})$ and that the aircraft can select from according to a decision strategy, is defined as the probability that a trajectory selected according to the strategy is feasible. For example, one decision strategy is to select a trajectory out of the total trajectories available at a point $(\mathrm{k})$ randomly (i.e. with equal probability) regardless of the outcome of disturbances. Using this simplified decision strategy, Idris showed that the probability of feasibility of the selected trajectory at any point $\mathrm{k}$ (i.e., RBT at point $\mathrm{k}$ ) can be estimated by the ratio of the expected number of feasible trajectories $\mathrm{f}(\mathrm{k})$ to the expected total number of trajectories $\mathrm{N}(\mathrm{k})$ available at the point $\mathrm{k} .{ }^{8} \mathrm{RBT}(\mathrm{k})=\mathrm{P}_{\mathrm{f}}(\mathrm{k})=\mathrm{f}(\mathrm{k}) / \mathrm{N}(\mathrm{k})$ where $\mathrm{N}$ is the sum of feasible and infeasible trajectories $\mathrm{N}(\mathrm{k})=\mathrm{f}(\mathrm{k})+\mathrm{i}(\mathrm{k})$ and the number of trajectories are expected values given the disturbance distributions.

2. Adaptability (ADP) is defined as the ability of the aircraft to regain feasibility of its trajectory in case it lost it due to the occurrence of disturbances. The aircraft regains feasibility by making another trajectory plan that is feasible by selecting from the feasible trajectories available to it at that point in space and time according to a decision strategy. Therefore, adaptability, denoted as ADP, at a point in space and time (k), is measured with the expected number of feasible trajectories $\mathrm{f}(\mathrm{k})$ available to the aircraft to select from according to a decision strategy, given the probabilities of the disturbances that pose the risk of constraint violation: $\operatorname{ADP}(k)=f(k)$.

\section{B. Discrete framework}

In order to measure robustness and adaptability as defined above, the number of total and feasible trajectories available to the aircraft at a point $\mathrm{k}$ in space and time to reach its destination needs to be estimated. The destination is a collection of points in space and time representing, for example, an RTA at a fix with some temporal or spatial tolerance. To be able to count the number of trajectories, a discrete representation of time and space and of the solution space of the aircraft trajectory is developed as follows: $:^{5-8}$

1. Time is discretized into equal time steps ( $\varepsilon$ apart) up to a time horizon T, representing for example the RTA.

2. The airspace available is discretized into rectangular cells in two or three dimensions, depending on the problem.

3. The aircraft degrees of freedom (speed, heading, and altitude) assume discrete values between maximum and minimum limits. Their limits and resolutions may represent operational procedures, human behavior, or decision strategy. For example speed may be allowed to change in increments of five knots only. The limits and resolutions may be different depending on the location in the time-space domain.

4. Each trajectory is assumed to consist of a time series of the discrete cells that are reachable given the aircraft degrees of freedom and the assumption that the values of the degrees of freedom are maintained constant between two successive time steps.

5. Reachability is measured from the centers of the cells, which are the points $\mathrm{k}$ at which the number of trajectories are estimated. A cell is considered reachable from a cell in the previous time step if any part of it is reachable given the assumption of constant values of the degrees of freedom. The number of ways a cell can reach another cell in the next time step are counted and recorded in the reachability function $\mathrm{g}(\mathrm{k})$. This is depicted on the left side of Fig. 4 for a two dimensional example with three heading and three speed values. The cells that are reachable from the center of a cell $(\mathrm{k})$ with coordinates $(11,11)$ over one time step are identified. The values of the function $\mathrm{g}(\mathrm{k})$ are noted on each cell that has a value larger than zero. The right hand side of Fig. 4 shows the 
process repeated for the centers of the reachable cells over the next time step. In this example the function $\mathrm{g}(\mathrm{k})$ was assumed the same for all cells, but it may be dependent on $\mathrm{k}$.
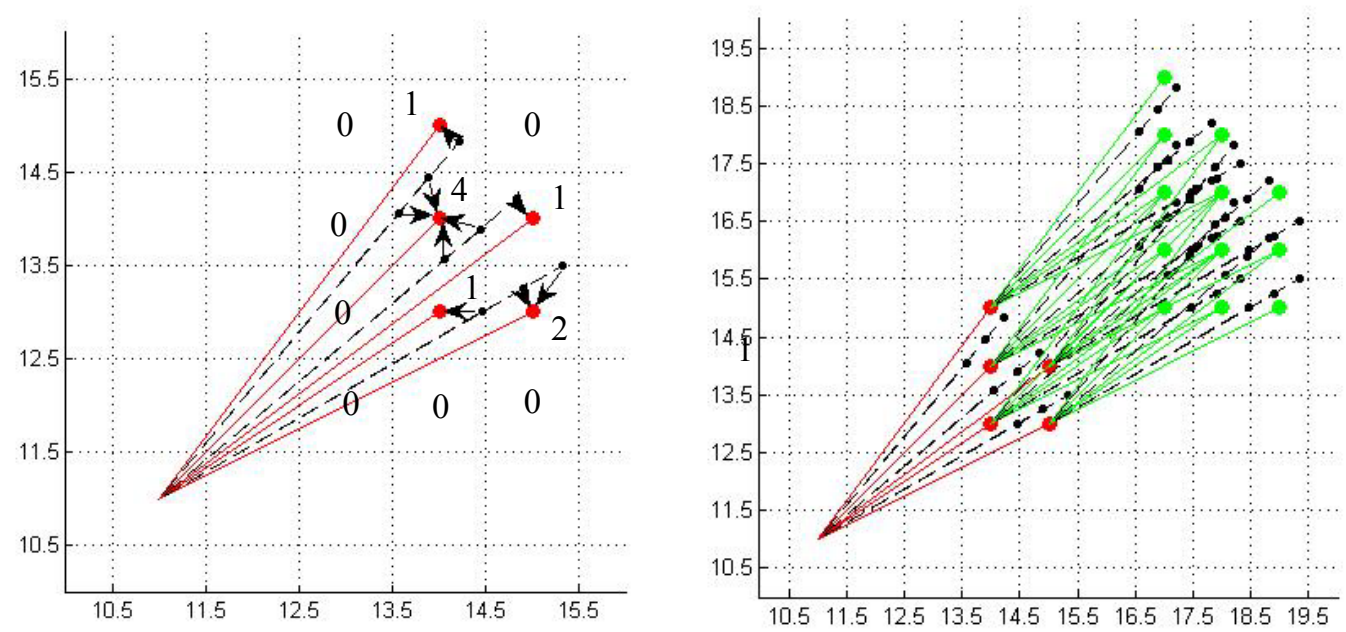

Figure 4. Example of discrete framework and reachability from one cell

6. The example shown in Fig. 4 is deterministic, where each of the reachable points that lie within a cell occurs with probability of one. To represent state disturbances, each of these points may be substituted with multiple possible reachable points with a probability value assigned to each. This value would scale the contribution of this point to the number of trajectories estimated according to the method described in the next subsection.

7. The number of trajectories is estimated at the center $(\mathrm{k})$ of each cell at each of the time steps. Therefore the size of the time steps and of the spatial cells represents the estimation sampling resolution.

\section{Estimation method}

A method for estimating the metrics has been devised, as described in Idris et al. ${ }^{5-8}$ The method estimates the number of trajectories that emanate from each of the cell centers $\mathrm{k}$ to the destination. It combines a convolution process to count the number of trajectories in the discrete reachability tree with a filtering process to eliminate infeasible trajectories that violate constraints. It is summarized in the following steps and depicted in Fig. 5 for a two dimensional case:

1. First, the last time step $T$ is initialized by setting the number of trajectories $f_{T}(k)$ to one for each cell $k$ that is feasible and to zero for each cell $\mathrm{k}$ that is not. Feasibility is determined based on constraint violation. If the center of the cell violates separation with another aircraft, or lies within a hazard airspace, or lies outside a required time-space region, then it is infeasible. In Fig. 5, the trajectory of an intruder traffic or hazard is enclosed in a polyhedral object (shown in red) that represents the separation requirements to ensure safety. The resulting polyhedral objects occupy certain volumes of the time-space domain. A center of a cell that lies inside the polyhedral object indicates infeasibility of the cell.

2. The reachability function $\mathrm{g}(\mathrm{k})$ is computed for each of the cells in the previous time step T-1. This results in $\mathrm{g}_{\mathrm{T}-1}(\mathrm{k} \rightarrow \mathrm{i})$ which is the number of trajectories that reach from each cell $\mathrm{k}$ in time step $\mathrm{T}-1$ to the cells $\mathrm{i}$ in time step T.

3. If the trajectory segment connecting the center of cell $\mathrm{k}$ in time step $\mathrm{T}-1$ and the center of cell $\mathrm{i}$ in time step $\mathrm{T}$ is not feasible, the corresponding value $\mathrm{g}_{\mathrm{T}-1}(\mathrm{k} \rightarrow \mathrm{i})$ is set to zero. Feasibility is also determined in terms of violating separation with another aircraft, crossing a hazardous airspace, or lying outside a required time-space region. In Fig. 5, it amounts to the segment crossing the polyhedral object representing an intruder aircraft trajectory.

4. Then the function $\mathrm{g}_{\mathrm{T}-1}(\mathrm{k})$ is multiplied by the function $\mathrm{f}_{\mathrm{T}}(\mathrm{k})$ of the last time step to compute the number of feasible trajectories $\mathrm{f}_{\mathrm{T}-1}(\mathrm{k})$ at each cell $\mathrm{k}$ in the previous time step: $\mathrm{f}_{\mathrm{T}-1}(\mathrm{k})=\sum_{\mathrm{i}}\left\{\mathrm{g}_{\mathrm{T}-1}(\mathrm{k} \rightarrow \mathrm{i}) \times \mathrm{f}_{\mathrm{T}}(\mathrm{i})\right\}$.

5. Steps 1 through 4 are then repeated for the previous time steps, replacing $T$ with $T-1$, and $T-1$ with $T-2$, until the initial state is reached. 


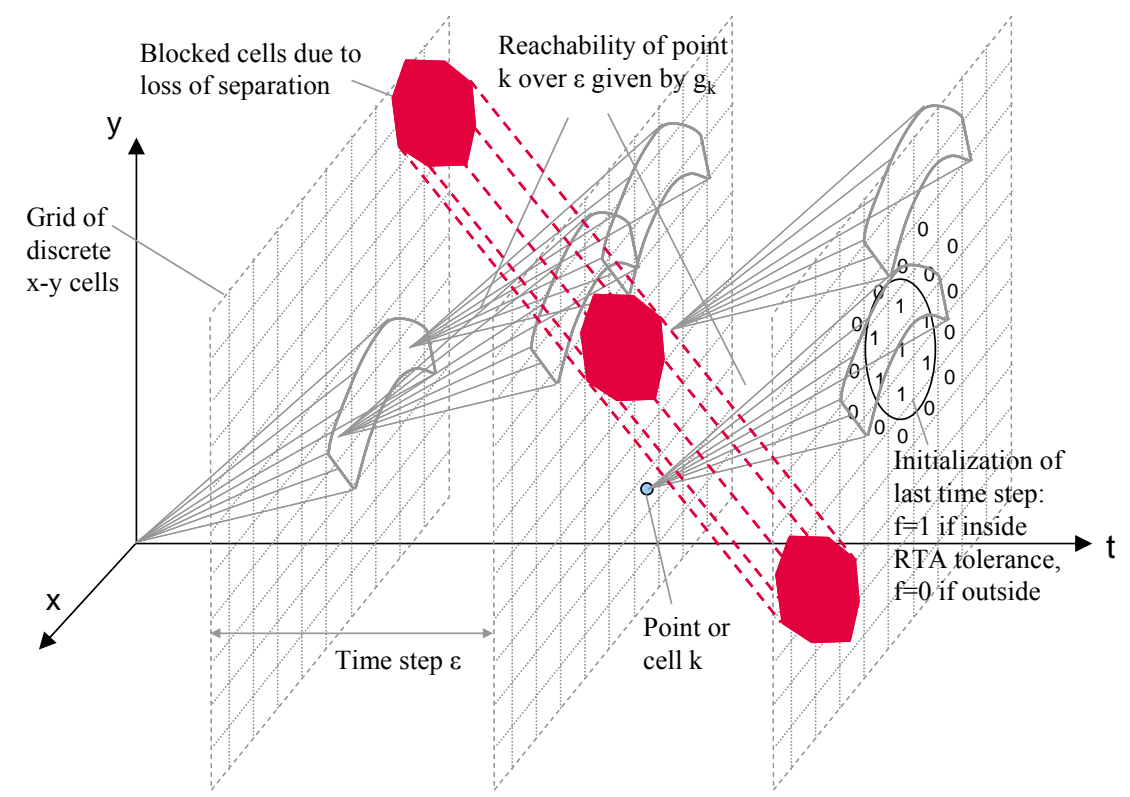

Figure 5. Discrete estimation of number of feasible trajectories

Note 1: In step 1, whether a cell is feasible or not was determined deterministically by setting the value of $\mathrm{f}$ to one if feasible or to zero if not. More generally, the value can be set to a fractional number between zero and one representing the probability of feasibility of the cell. For example, if the cell is impacted by a weather cell and the impact is represented by a probability of occurrence, this probability can be used. Similarly, the values of $\mathrm{f}$ computed in the previous time steps can be scaled by a number between zero and one, representing the probability of feasibility of the cells in these time steps. The analyses in the following sections of this paper are deterministic.

Note 2: In step 3, the number $g_{t}(k \rightarrow i)$ was set to zero if the trajectory segment that reaches from the center of cell $\mathrm{k}$ to the center of cell $\mathrm{i}$ between the time steps $\mathrm{t}$ and $\mathrm{t}+1$, is not feasible. This requires testing only the segment between the two centers for feasibility. Alternatively, each of the segments that reach from the center of cell $\mathrm{k}$ and reaches anywhere within the cell i over the time step from $t$ to $t+1$ (see Fig. 4) can be tested for feasibility. Then the number $\mathrm{g}_{\mathrm{t}}(\mathrm{k} \rightarrow \mathrm{i})$ can be reduced by the number of segments that are not feasible. While this would result in a more accurate estimate of the number of feasible trajectories, it requires more computation. In the analysis in this paper, the first method of testing the segments connecting the centers only is used.

\section{Flexibility metric alternatives}

Given the discrete representation of the trajectory solution space, a number of metrics can be formulated to measure RBT, ADP, or combinations of them. The following metrics are considered:

(1) Time duration of the trajectory is a measure of robustness to unknown disturbances. Since there is no information about unknown disturbances, one may assume that all points in space and time have equal probability of feasibility with respect to unknown disturbances, say equal to $\mathrm{P}_{\mathrm{f}}$. The probability of feasibility of a trajectory may be obtained by multiplying the independent probabilities of feasibility along it, as described in subsection A above. If these probabilities are all independent and equal to $\mathrm{P}_{\mathrm{f}}$, then the probability of feasibility of the trajectory is $\mathrm{P}_{\mathrm{f}}($ traj$)=$ $\left(\mathrm{P}_{\mathrm{f}}\right)^{\mathrm{T}}$ where $\mathrm{T}$ is the time duration of the trajectory. Therefore, in order to maximize robustness with respect to unknown disturbances, the time duration of the trajectory $\mathrm{T}$ should be minimized. If an aircraft is required to reach a destination point at a specific time, all its possible trajectories are equivalent in terms of their robustness to unknown disturbances because they have the same duration.

(2) One extreme measure of robustness is the probability of feasibility assuming random selection of solutions for the degrees of freedom at each decision point, without making any attempt to adapt to the outcome of the disturbances. Equivalently, the aircraft degrees of freedom are decided by totally random state disturbances at each decision point. This assumption makes all possible values of the degrees of freedom, and hence trajectories, equally likely at each decision point. Consequently this extreme measure of robustness can be easily calculated at each point in time and space as the ratio of the number of feasible trajectories to the total number of trajectories at that point as described for RBT in subsection B above. 
(3) Another extreme measure of robustness is the probability of feasibility assuming that the aircraft makes a decision to adapt to the outcome of the disturbances and avoids the infeasibility at each decision point, unless it is not possible because a feasible option is not available. This assumption requires timely and perfect information about the disturbances before acting, which is not always possible. This form of robustness is the complement of the probability that the disturbance outcome leaves no feasible option to be used by the aircraft to adapt. Less extreme measures are also possible. For example, one may measure the probability of having at least ' $x$ ' number of feasible options or having ' $\mathrm{x}$ ' percent of the total options being feasible to use to adapt at every decision point. Unlike the measure of robustness under the random decision making strategy in (2), this measure requires knowledge of the probability distributions of the disturbances to calculate the probability of feasibility.

(4) The two situations (2) and (3) are extremes, the first representing total lack of information and no ability to adapt and the second representing timely information and perfect ability to adapt. The difference between them is the contribution possible to robustness by added information or by added adaptability. Hence the difference may be considered a measure of the need to adapt due to the lack of perfect information and decisions.

(5) Adaptability is measured, as described in subsection B, by the expected number of feasible trajectories that are available to the aircraft at a point to adapt to disturbances that makes its current trajectory infeasible, given the probability distributions of these disturbances. Adaptability measures the ability to react to unforeseen disturbances as well as the ones partially known. The assumption is that the best planning strategy is to maintain the highest number of expected feasible trajectories at each point along the trajectory to react to any disturbance. Adaptability of a trajectory may be measured, therefore, as the summation of the adaptabilities along the trajectory.

In summary, a number of metrics may be used, separately or in combination, for mitigating the exposure to the risk of constraint violation by an airborne or a ground-based control system:

1. Reduce the duration of a trajectory to increase robustness to unknown disturbances (1).

2. Increase the robustness to partially known disturbances, conservatively assuming no adaptability (2) and less conservatively assuming full or partial adaptability (3).

3. Increase robustness by reducing the need to adapt to partially known disturbances (4).

4. Increase the expected number of feasible trajectories available at each point along the trajectory to increase the ability to adapt to any disturbances (5).

\section{E. Trajectory planning using flexibility metrics}

Given the discrete structure of the solution space, dynamic programming offers a straightforward method to build an optimal trajectory. Using recursive back-propagation and starting from the final time step, the minimum cost of proceeding from each cell to the destination is computed and stored. This minimum cost $Q(t, x(k), y(k))$ for each cell $\mathrm{k}$ is computed by minimizing, over its reachable cells in the next time step $\mathrm{t}+1$, the sum of the minimum cost $\mathrm{Q}(\mathrm{t}+1, \mathrm{x}, \mathrm{y})$ already computed for each of the reachable cells $(\mathrm{t}+1, \mathrm{x}, \mathrm{y})$ plus the cost of proceeding from $\mathrm{k}$ to that cell, given for short by $\mathrm{q}(\mathrm{k} \rightarrow(\mathrm{t}+1, \mathrm{x}, \mathrm{y}))$. A generic formula is:

$$
\mathrm{Q}(\mathrm{t}, \mathrm{x}(\mathrm{k}), \mathrm{y}(\mathrm{k}))=\operatorname{Min}_{\mathrm{x}, \mathrm{y}: \mathrm{g}_{\mathrm{k}}(\mathrm{t}+1, \mathrm{x}, \mathrm{y})=1}\{\mathrm{Q}(\mathrm{t}+1, \mathrm{x}, \mathrm{y})+\mathrm{q}(\mathrm{k} \rightarrow(\mathrm{t}+1, \mathrm{x}, \mathrm{y})\}
$$

Four functions for the local cost, q, were used in the experiments reported in this paper. A function representing minimal path length was used as a baseline. Functions representing maximizing adaptability, maximizing robustness, and maximizing both combined with minimizing path length:

$$
\begin{aligned}
& \mathrm{q}(\mathrm{k} \rightarrow(\mathrm{t}+1, \mathrm{x}, \mathrm{y}))=\operatorname{distance}(\mathrm{k} \rightarrow(\mathrm{t}+1, \mathrm{x}, \mathrm{y}))=\text { dist } \\
& \mathrm{q}(\mathrm{k} \rightarrow(\mathrm{t}+1, \mathrm{x}, \mathrm{y}))=-\operatorname{ADP}(\mathrm{k}) \\
& \mathrm{q}(\mathrm{k} \rightarrow(\mathrm{t}+1, \mathrm{x}, \mathrm{y}))=-\operatorname{RBT}(\mathrm{k}) \\
& \mathrm{q}(\mathrm{k} \rightarrow(\mathrm{t}+1, \mathrm{x}, \mathrm{y}))=-\operatorname{ADP}(\mathrm{k})-\mathrm{a}^{\mathrm{T}} \operatorname{RBT}(\mathrm{k})+\mathrm{b}^{\mathrm{T}} \text { dist }
\end{aligned}
$$

where $\mathrm{a}$ and $\mathrm{b}$ are weights that trade robustness and distance, respectively, with adaptability. They are raised to the power of time $\mathrm{T}$ (measured from the final time step) to account for the exponential growth of ADP. Note that while the accumulated distance over time is minimized, ADP and RBT are maximized at each time step (because their accumulation at any point is identical over all trajectories to the destination). After storing the optimal costs for each cell, a forward loop builds a trajectory by tracing the optimal cells starting from the initial state. Any ties between cells are broken randomly.

Figure 6 shows an example of the discrete solution space in two spatial dimensions and time, and one trajectory (black line) selected through the solution space using adaptability on the left and robustness on the right hand sides 
respectively. Using color coding, the cost function value using ADP is shown on a log scale on the left hand side (because ADP grows exponentially with negative time) while the cost function using RBT is shown on the right hand side. Note that while RBT is between zero and one at each cell, the cost function is cumulative over time hence the numbers grow larger than zero. Note also the white regions within each grid in each time step. These white regions are the infeasible regions due to trajectories of surrounding traffic or hazards, and the values in them are set to zero.

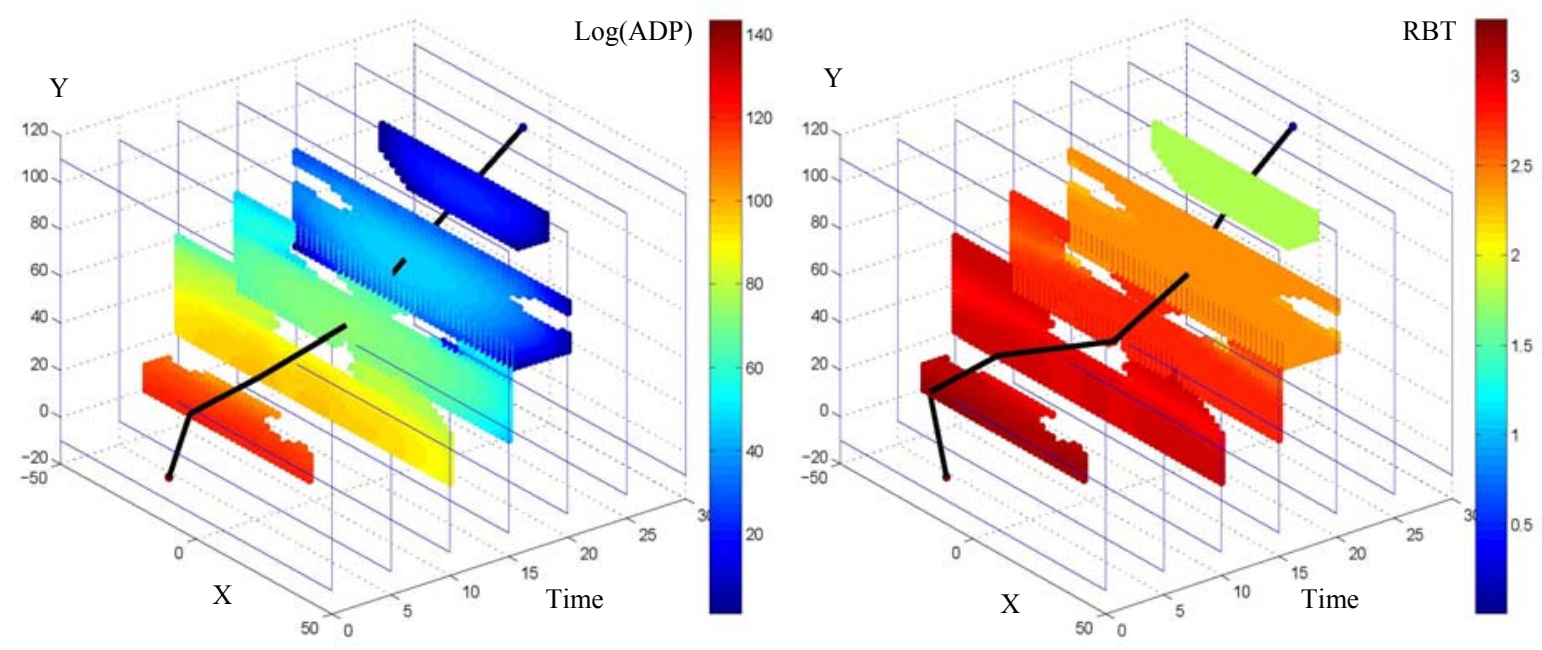

Figure 6. Example of the discrete solution space and ADP and RBT map along with a trajectory solution

\section{Traffic Complexity Management}

In this section, the flexibility metrics presented in the previous section are applied to the problem of traffic complexity management. The hypothesis is that, by airborne or ground control systems applying these metrics to plan aircraft trajectories such as to mitigate the exposure of each aircraft to the risk of constraint violation, traffic complexity is naturally reduced. The hypothesis was tested in a distributed application, where each aircraft independently applies the metrics to plan its trajectory without coordination. The metrics used in these analyses were robustness as explained in (2) in subsection II.D and denoted RBT and adaptability as explained in (5) in subsection II.D and denoted by ADP. Many approaches have been documented to define and measure traffic complexity, most often as a function of controller workload. These metrics are primarily based on airspace geometry such as aircraft density and mix, sector geometry, traffic flow structure, and mix of aircraft types and performance characteristics. ${ }^{12-}$ ${ }^{14}$ Other efforts emphasized cognitive elements of complexity, in particular the controller use of standard flows, grouping of traffic, and merge points. ${ }^{15}$ Some metrics have been proposed that are independent of the airspace structure and controller perspective. For example, Delahaye et al. introduced complexity metrics based on traffic organization or disorder (topological entropy). ${ }^{16}$ A number of these traffic complexity indicators were analyzed to test this hypothesis in simple scenarios as described in the following subsections.

\section{F. Traffic pattern consistency}

It was observed that the use of ADP, RBT, or a combination of the two to plan aircraft trajectories resulted in traffic patterns that were more consistent, relative to using other metrics such as the shortest path. This was tested using two dimensional scenarios that involved aircraft making decisions to go through holes between weather cells or to travel around a roundabout. ${ }^{9,10}$ The roundabout scenario is shown in Fig. 7. It consists of a weather cell that causes four traffic flows crossing at right angles to go around it in a roundabout. The weather cell is modeled as a circle located at the center of a square region of airspace. Each flow originates at a middle point of an edge of the square (triangles) and ends respectively a short distance before the middle point of the opposite edge (crosses). Eight other hazard circles are added at the corners to increase the traffic interaction around the hazard located in the center. All aircraft are limited to headings of \pm 60 degrees relative to the centerline connecting the start and end positions, with ten-degree increments. They are also limited to a speed between 240 and 360 knots with ten-knot increments. Each aircraft plans a trajectory to meet an assigned RTA (using speed reduction and path stretching), 
while optimizing a metric such as ADP, RBT, or shortest path. The first aircraft does not encounter any traffic and is only affected by the hazards. Then each following aircraft plans its trajectory under the impact of the trajectories of the preceding aircraft and the hazards which reduce the number of feasible trajectories as described in Section II. Time increments of two minutes and square x-y cells of two nautical miles are used in the estimation of the number of trajectories. The experiment considered only deterministic aircraft behavior, where one trajectory was considered for each aircraft with probability of one and was not updated dynamically. However, the separation requirement around each aircraft was set to ten nautical miles (instead of the required five nautical miles) in order to capture the higher uncertainty in the rather long time horizon of the experiment.

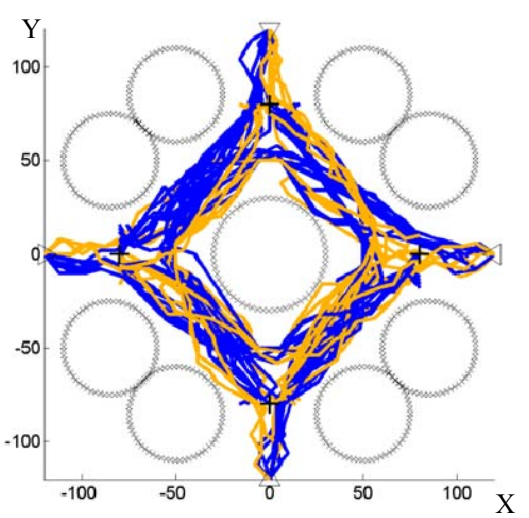

Shortest path with traffic avoidance

Pattern: $68 \%$ of aircraft counterclockwise (blue)

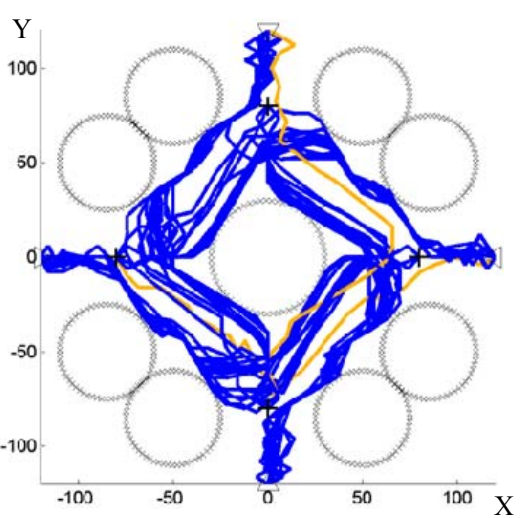

Maximum adaptability only

Pattern: $97 \%$ of aircraft counterclockwise (blue)

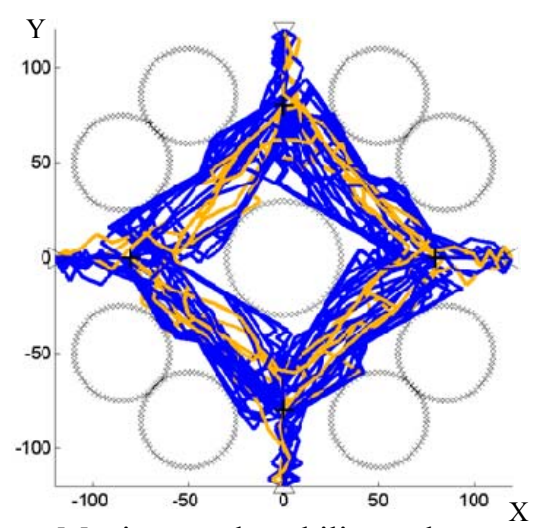

Maximum adaptability and robustness, and shortest path

Pattern: $84 \%$ of aircraft counterclockwise (blue)

Figure 7. Flow patterns in roundabout scenario

Figure 7 demonstrates the traffic patterns that resulted by plotting the full trajectories starting at the sources and ending at the sinks. Color is used to distinguish the direction relative to the central hazard. Most aircraft turned around the central hazard in a uniform direction relative to the shortest path case. This is indicated in the figure by the percentage of aircraft that selected the counterclockwise direction (using the darker, blue color). This percentage is 80-97 percent when ADP or RBT are used alone or in conjunction with shortest path compared to 68 percent when shortest path is used alone while avoiding the traffic and hazards. Not shown in Fig. 7 are the cases of robustness alone which resulted in 70 percent counterclockwise traffic and shortest path without avoiding other traffic which resulted in only 60 percent counterclockwise traffic.

\section{G. Traffic flow entropy}

A Lyapunov exponent metric which measures the level of entropy in a traffic flow field, was used as the traffic complexity indicator. ${ }^{9}$ The analysis was conducted using the two-dimensional scenarios described in the pattern consistency analysis described in the previous subsection. It was demonstrated that the Lyapunov exponent was consistently improved when ADP, RBT, or a combination of the two were used in trajectory selection compared to selecting the shortest path. This is shown in Fig. 8 in two ways. In the top three plots, the Lyapunov exponent values for the three plots in Fig. 7 are shown for one instance of time as the background color. Blue background color indicates low exponent values (low entropy) while green and red flashes indicate regions of high values (i.e., high entropy). The low entropy indicates more traffic organization which corresponds to lower gradients in the speed vector field. On the other hand, high entropy values indicate high gradients corresponding to less organized traffic and hence higher uncertainty in predicting the flow. These snap shots indicate that lower entropy and hence higher predictability resulted when ADP or RBT were used relative to the shortest path as objectives. When ADP or RBT were used, the background was predominantly blue, except around the source and destination points. On the other hand, in the shortest path case, there are more green regions around the central hazard because aircraft more often travel in opposite directions as demonstrated in the previous subsection. The plot in the lower part of Fig. 8 shows the aggregate value of the Lyapunov exponent over time. It indicates that when ADP and RBT were used (and in particular ADP), the entropy was lower during most of the run. 


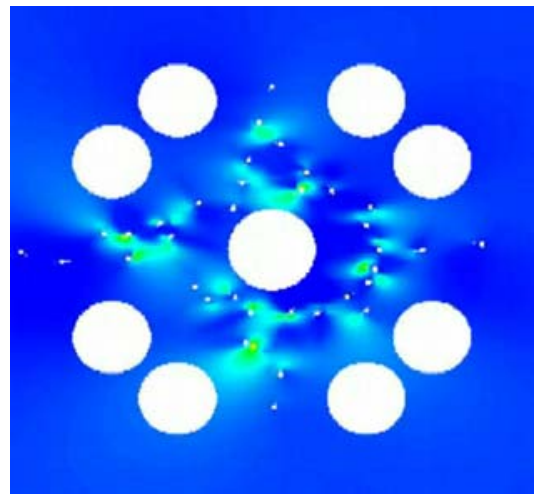

Shortest path with traffic avoidance

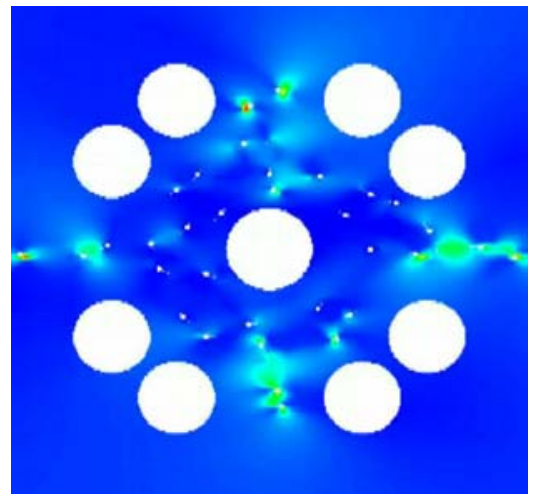

Maximum adaptability only

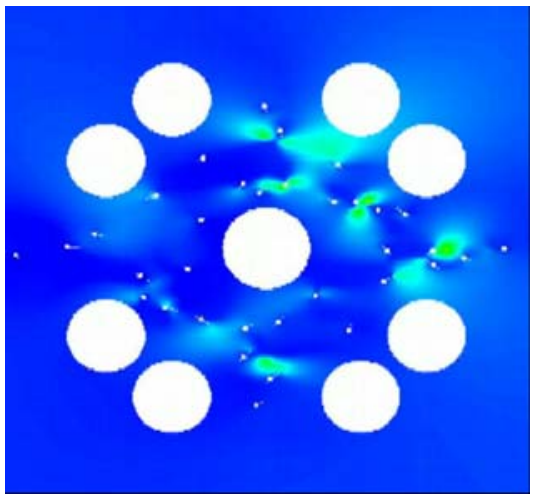

Maximum adaptability and robustness, and shortest path

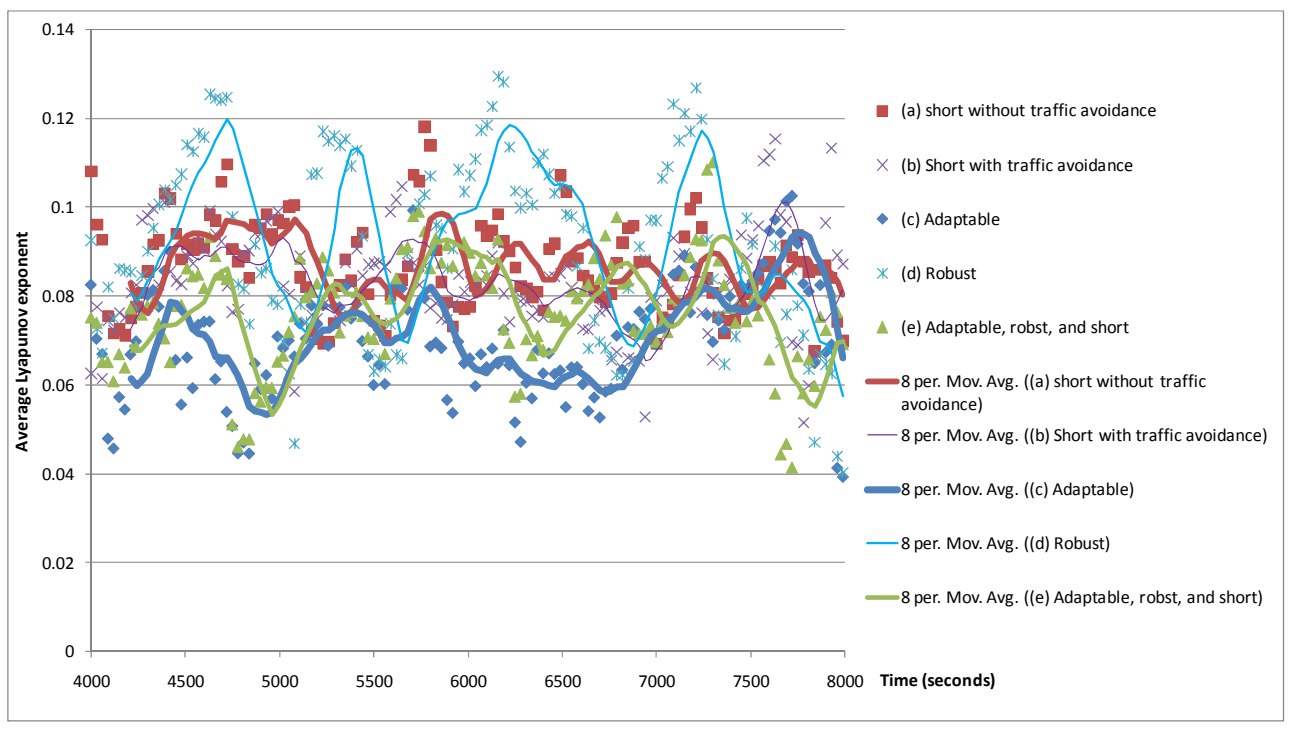

Figure 8. Lyapunov exponent in roundabout scenario

\section{H. Dynamic density}

Six dynamic density indicators were used as traffic complexity metrics to test the results of the same scenarios. ${ }^{10}$ The indicators were: traffic density, traffic proximity, relative heading, number of impending conflicts (predicted loss of separation), relative headings of impending conflicts, and time to predicted loss of separation. For all these indicators, it was demonstrated that the use of ADP, RBT, or a combination of the two resulted in mitigating traffic complexity relative to selecting the shortest path. Four examples are shown in Fig. 9. The top left plot compares traffic density measured by the number of cells that were occupied by more than three aircraft over the run (where the area of the scenario is divided into 64 square cells). It shows that the lowest traffic density resulted when ADP was used alone or combined with robustness. The top right plot shows similar results for proximity, which was measured as the inverse of a weighted horizontal separation between aircraft (higher weight is given to closer proximity, according to the dynamic density indicator). The lower left plot shows that the relative headings of aircraft had less variation when adaptability was used, and finally the lower right plot shows the reduction of the number of impending conflicts, again particularly when ADP was used. 

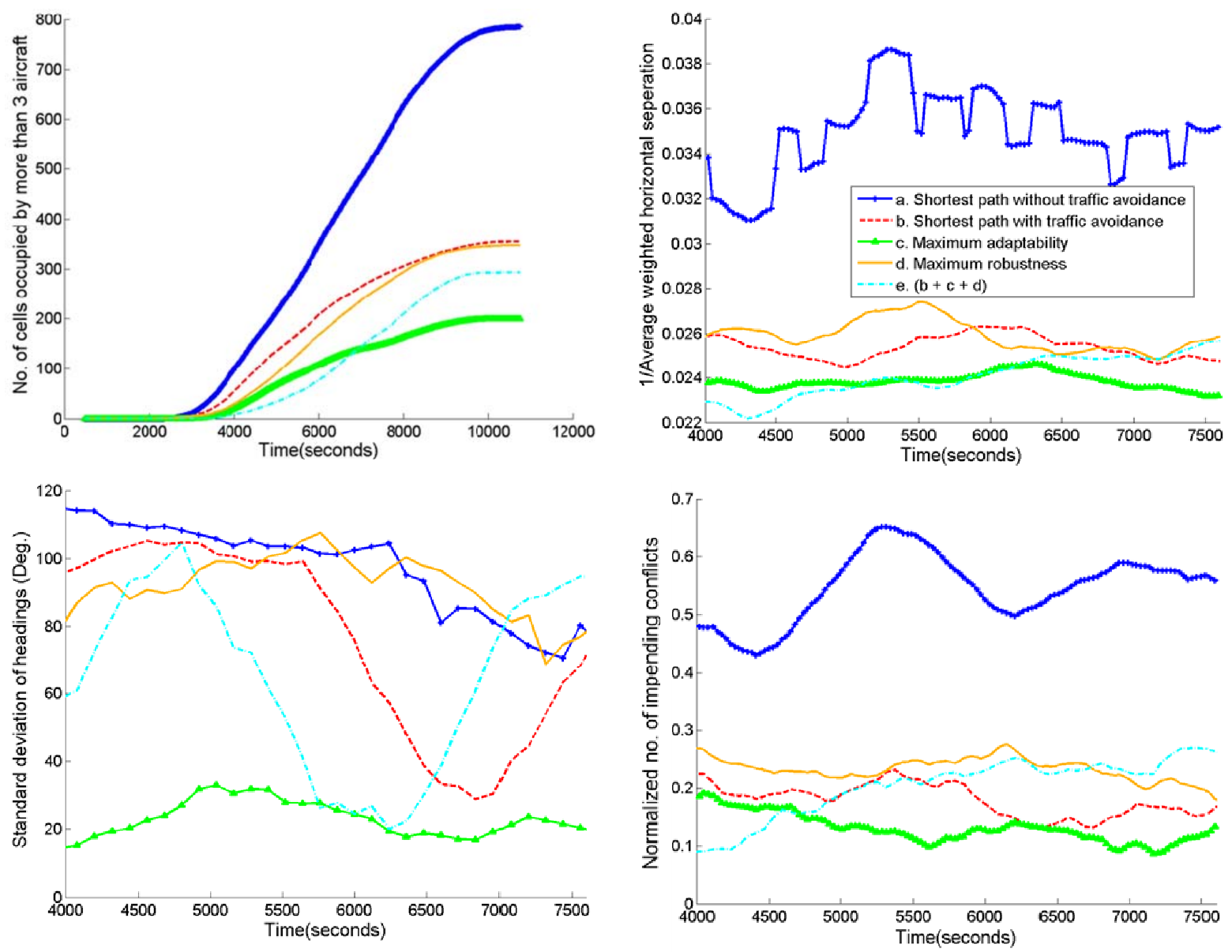

Figure 9. Dynamic density analysis of roundabout scenario

\section{Use of altitude}

A three dimensional scenario was analyzed to test the impact of using the altitude degree of freedom in addition to speed and heading on mitigating traffic complexity. A number of simplifications were made in extending the metrics and their estimation technique to the altitude degree of freedom. Most notably: (1) The altitude was allowed to vary less frequently than the heading and speed degrees of freedom, at decision points that are multiples of the ones allowed for heading and speed. This simplification is operationally motivated in order to avoid frequent altitude changes. (2) The altitude was assumed to change instantaneously ignoring the climb and descent dynamics. This simplification is computationally motivated and is reasonable given the long-horizon planning considered in this scenario where modeling the dynamics accurately is not critical for the altitude selection decision. (3) The traffic and hazards are assumed to be contained within each altitude level for simplicity.

The scenario consisted of six aircraft, numbered one through six in Fig. 10. Two altitude levels are allowed denoted as 3000 and 6000 in the figure. Each aircraft starts at the top level from the point where the aircraft number is shown in the figure and travels to a destination point along a straight line to the opposite side. This geometry makes aircraft one and two, aircraft three and four, and aircraft five and six, head towards each other. Each aircraft is assigned an RTA at the destination point but no altitude restriction so it can arrive at either level. The aircraft can avoid each other and meet their RTA using altitude, path stretching, and speed, where the altitude is allowed to change every ten speed/heading decision points. Figure 10 shows the analysis of how the altitude, heading and speed were traded off. The top two plots show the three dimensional trajectories of the six aircraft maximizing ADP on the left side and maximizing RBT on the right side. The second set of plots show the corresponding lateral deviations comparing using ADP on the left to using RBT on the right. The third row contains a plot on the left that compares the average speed used for ADP and RBT and a plot on the right that shows the distribution of aircraft on the two altitude levels, which was identical for the ADP and RBT cases. As shown in the three dimensional trajectories, the first aircraft remained at the top altitude level since no other aircraft exist when it planned its trajectory. Having the 
altitude degree of freedom as an additional option allows the second aircraft to descend to the lower altitude level because aircraft one occupies the higher altitude. The other aircraft, three through six, make decisions to alternate between the two altitudes as optimal from the adaptability or robustness perspectives, each in reaction to the previous ones. As shown in the lower right plot in Fig. 10, the aircraft use both levels converging to an equal distribution of three aircraft per level, for both ADP and RBT. This indicates that ADP and RBT both resulted in using the two altitude levels effectively to reduce the congestion in each. The lateral deviation plots show that, as expected, using ADP does not result in large lateral deviation, keeping the trajectory near the line of sight for each aircraft where there are more trajectory options. On the other hand, using RBT tends to send the aircraft towards the extremities of the solutions space as far from the other traffic as possible, since RBT is measured with respect to violating separation from the traffic in this scenario. Since the aircraft are required to meet an RTA at the destination point, the average speed plot shows lower speed when ADP is used than when RBT is used. This is expected since higher speed is needed to compensate for the larger lateral deviation when using RBT relative to using ADP.
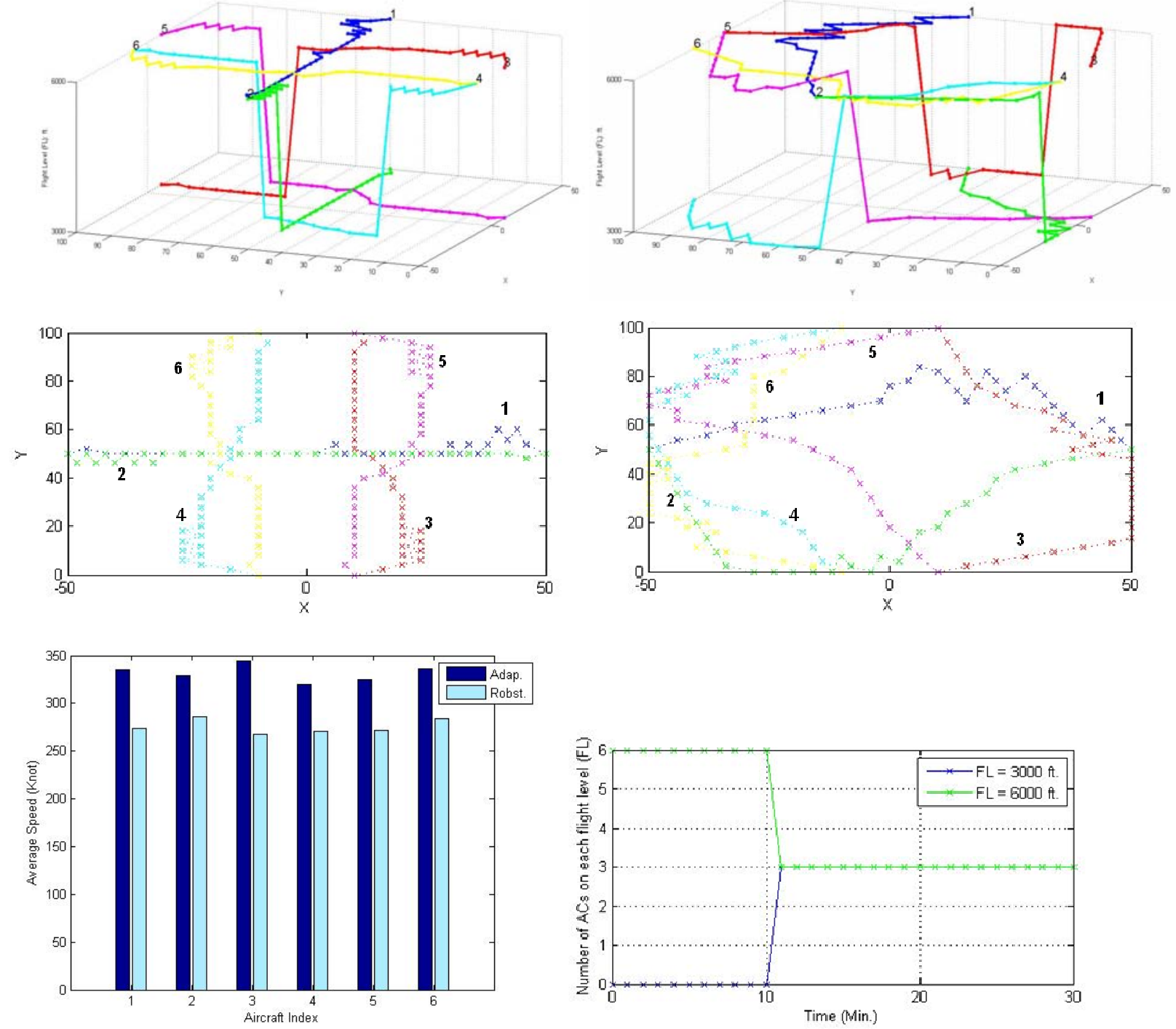

Figure 10. Analysis of using altitude versus heading and speed

\section{J. Secondary conflicts}

As described in Section I, preserving trajectory flexibility in conjunction with maintaining separation between aircraft may induce implicit coordination between the aircraft that are being separated. For example, when each aircraft selects a conflict free trajectory that also reduces the risk of exposure to disturbances from the other traffic, 
the risk of secondary conflicts may be reduced. This is particularly useful in distributed separation assurance where aircraft are delegated the authority to separate from other traffic and where implicit or explicit coordination between aircraft actions is needed. Reducing the risk of secondary conflict would also benefit centralized ground-based separation assurance by improving the stability of the aircraft trajectories.

In Idris et al, this hypothesis was tested in a simple scenario similar to the one described in the previous subsection in two dimensions. ${ }^{11}$ The six aircraft shown in Fig. 11 generated trajectories simultaneously and independently without any coordination, assuming knowledge about the initial trajectories of the other aircraft but nothing about their intensions. The resulting fifteen trajectory pairs (for each two aircraft of the six) were tested for secondary conflict. When robustness and adaptability were used in the objective functions the potential of secondary conflict was significantly reduced, relative to using the shortest path as an objective. In over 15000 trials, on average 5.8 of the fifteen pairs resulted in secondary conflict when the shortest path was used compared to 5 of the 15 when adaptability was used and 4.28 of the fifteen when robustness was used. The means are significantly different at the 0.01 level. The histograms are depicted in Fig. 11 along with ten trajectories for each aircraft in each case. Although adaptability showed some improvement, robustness was more effective at reducing the potential for secondary conflicts. This is expected because robustness, measured by the ratio RBT, in this case measured the exposure to violating separation from the other traffic explicitly. On the other hand, adaptability measured by the number of feasible trajectories attempts to increase the ability to respond to any disturbances, including the ones by the existing traffic but also unknown disturbances, such as pop up traffic or other hazards. The trajectories and histograms in Fig. 11 also show that the variability of the trajectories produced by adaptability is much lower. This is because the number of feasible trajectories ADP is much more distinct for each point in the solution space, while the ratio RBT is unchanged at many points (for example, equal to one), resulting in ties in the objective function that are resolved randomly. The same is also true of the shortest path case.
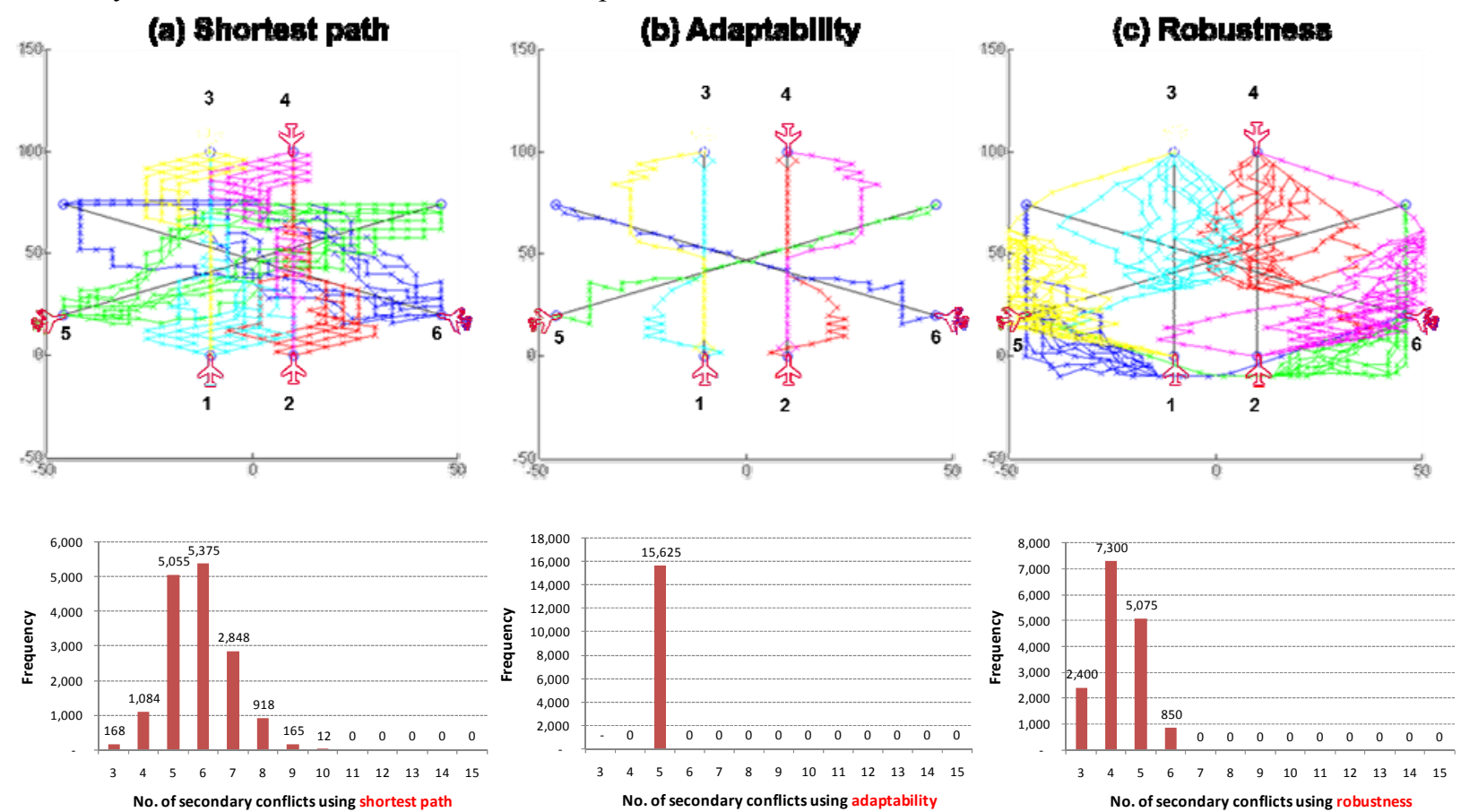

Figure 11. Analysis of secondary conflicts

\section{Constraint Minimization}

The service provider is responsible for maintaining safe and efficient airspace operations. Under trajectory-based operations, this is achieved largely by imposing constraints on aircraft trajectories. One major process by which trajectory constraints are generated is flow management, which includes identifying alternative routes for flights and defining rate restrictions to maintain demand for airspace resources below capacity. In delegated or self-separation schemes, where the pilot is responsible for safety and the service provider does not directly control the aircraft's trajectory to provide separation assurance, applying trajectory constraints on the autonomous aircraft is the primary means for the service provider to maintain efficient airspace operations. To enable an adequate amount of aircraft 
autonomy, service provider-generated trajectory constraints should only be applied when necessary and should be designed to apply the minimum level of constraint on the aircraft's trajectory that maintains efficient airspace operations.

\section{K. Trajectory constraint types}

The ground system and user Flight Operations Center impose a range of trajectory constraints to aircraft, e.g., from tactical heading clearances to route changes. While no attempt is made to comprehensively identify all types of trajectory constraints that may be imposed, two categories are distinguished, strategic and tactical trajectory constraints. In this context, strategic trajectory constraints are defined as those that can be applied directly to the routes within the aircraft's flight management system. To ensure increased flexibility, it is recommended that only strategic trajectory constraints be applied to aircraft with delegated or self separation. Tactical trajectory constraints might compromise the aircraft's ability to fulfill its requirements to maintain self-separation. On the other hand, strategic trajectory constraints may be applied with tolerance limits and are likely to be a candidate for relaxation. Examples of strategic constraints are:

1. Crossing restrictions, for example, crossing a fix at a specific time absolute or relative to another aircraft; at, below or above a certain altitude; and at, above or below a certain speed.

2. Path specifications, for example, cruise altitude or staying along a route segment.

3. Airspace restrictions, for example, from entering an airspace volume within certain time limits.

4. Traffic separation, for example, the minimum required separation for safety, but also including spacing intervals that are higher than the minimum requirements typically imposed for flow management.

\section{Constraint negotiation}

Negotiation to relax (i.e., eliminate or modify to make less restrictive) a service provider generated trajectory constraint can be desired or required by the flight crew under a variety of situations. Three situations are described:

(1) Overly constrained: The flight crew may not be able to accommodate all trajectory constraints issued to the aircraft. In this situation, the aircraft is said to be overly constrained. This may be due to performance limitations of the aircraft or inability to maintain self-separation while achieving the trajectory constraints.

(2) Excessively constrained: The flight crew may determine that the trajectory constraints issued can be met, but that the solutions available are not sufficiently flexible i.e., solutions do not maintain the risk of trajectory constraint violation below an acceptable level and that a future disturbance is likely to make the trajectory constraints unachievable. In this situation, the aircraft is said to be excessively constrained.

(3) Undesirably constrained: The flight crew may determine that the trajectory constraints issued can be met, but at the expense of other user objectives. A number of user objectives lead to various causes of undesirability, such as excessive fuel burn or travel time.

These situations result in requests from the air to the ground system to relax one or more trajectory constraints. The overly constrained situation results in an "Unable" message indicating that the aircraft is unable to meet all of the imposed trajectory constraints. The excessively constrained and undesirably constrained situations result in airborne requests for relaxing trajectory constraints; however, the excessively constrained message may be more important than the undesirably constrained one from both the user and service provider perspectives. The unable message is the most important message requiring service provider attention. In the following subsection the use of the trajectory flexibility metrics in assessing the overly and excessively constrained situations is discussed.

\section{A.A.1 Unable message for overly constrained situation}

The overly constrained situation may be determined when the aircraft automation is unable to find a feasible trajectory that meets all of the imposed trajectory constraints. In generating such a trajectory, the aircraft automation will include trajectory constraints that are imposed by the ground system as well as limits imposed by the airborne system, the violation of which are considered unacceptable. For example, the constraints may include aircraft performance limits. They may also include operational limits such as violating flight crew time legality. 


\section{A.A.2 Consideration of trajectory flexibility for excessively constrained situation}

In order to decide if an aircraft is excessively constrained, an absolute measure of flexibility, with a threshold for the condition of being excessively constrained, is needed. The flexibility metric needs to measure flexibility at the current and future states given the solution space available and potential state and constraint disturbances.

The metrics proposed in Section II were used to compare the flexibility available in the solution space under different trajectory constraint schemes. The suitability of the different metrics for this evaluation varies. Figure 12 shows the impact of varying the tolerance around an RTA on the adaptability and robustness of a single aircraft. The scenario is that presented in Section III with six aircraft, where one of the six aircraft, that is planning its trajectory in the presence of the other aircraft, is used as an example here. The tolerance is in terms of a distance radius allowed around the destination point, which is to be met at thirty minutes from the current time. The figure shows ADP and RBT over the optimal trajectory plan when maximizing ADP or RBT under different values of the RTA tolerance. The figure shows that, as expected, ADP increases as the tolerance is increased, because the number of trajectories available in the solution space increases. ADP is normalized with respect to the lowest tolerance analyzed. The impact on RBT is similar to the impact on ADP, with RBT increasing as tolerance increases. Robustness in this example was measured with respect to meeting the RTA as well as avoiding the other traffic.
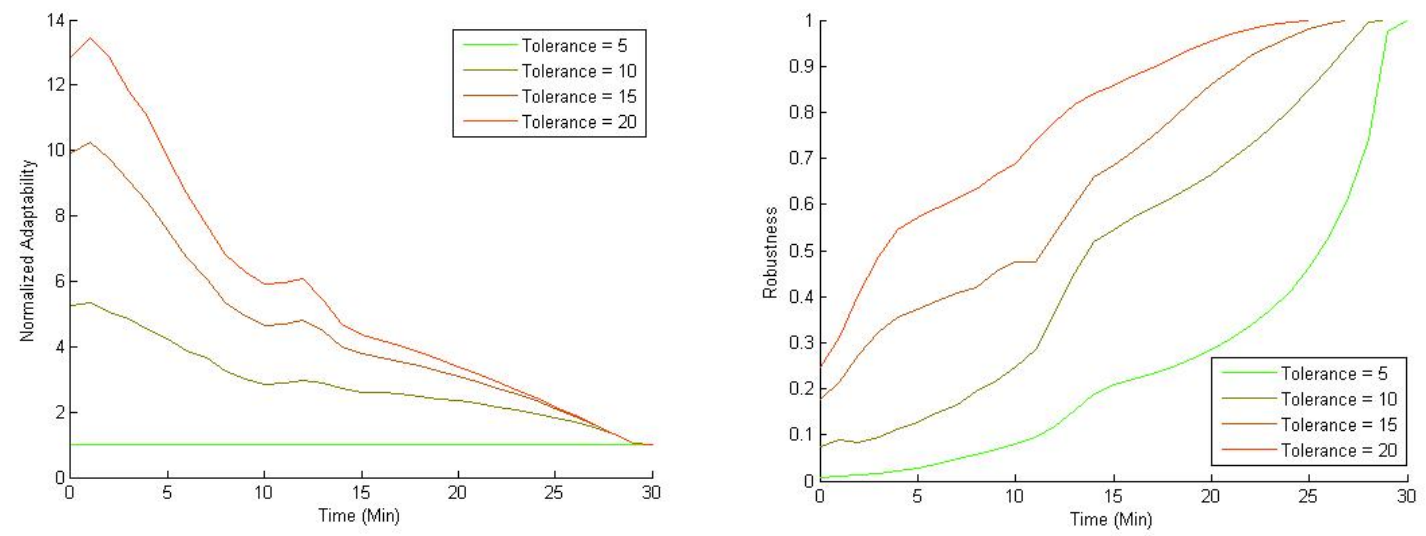

Figure 12. Impact of RTA tolerance on adaptability

ADP measures adaptability to all disturbances, including unknown ones, because it tries to keep as large a number of feasible trajectories as possible to deal with any disturbance situation. However, ADP suffers from being a large number in absolute terms because it grows exponentially, and the number depends on the discrete representation used for the estimation. ADP is not normalized and hence it is hard to set a threshold on it. Having more feasible trajectories is better but how many feasible trajectories is an acceptable number is a difficult question to answer. On the other hand RBT is normalized between zero and one, so it is easier to set a threshold on it based on the risk attitude of the airborne or ground decision maker. However, it measures the robustness with respect to violating partially known constraints and is dependent on the choice of the constraints with respect to which the robustness is measured. Therefore, if RBT is to be used to assess the risk exposure, a choice has to be made about the constraints with respect to which the risk of violation is measured. In this example, robustness was measured with respect to maintaining separation from the other traffic as well as violating the RTA constraint. Relaxing the RTA constraint in this case resulted in less exposure to violating both traffic and RTA constraints. One may chose to use robustness with respect to only separation from the traffic, or only meeting the RTA, or to both, but with different weights assigned to each if, for example, maintaining separation is more critical than meeting the RTA.

The RBT measure used in Fig. 12 assumes no attempt is made to adapt to disturbances. That is, the decision at each decision point is made arbitrarily, which makes it is easy to compute. The other metrics discussed in Section II may also be considered for the assessment of constraint excessiveness; however, they have not been analyzed due to the difficulty in computing them, which requires knowledge of the probability distributions of disturbances. For example, RBT may be computed using the extreme measure of robustness described in item (3) of Section II.D which measures the robustness to constraints assuming full ability to adapt. In this case RBT would be the probability of having at least one feasible option to adapt in every disturbance situation. In the example described in this section, its value would be equal to one since the traffic is sparse and there is at least one feasible option in 
every disturbance situation. Less extreme measures of robustness assuming some level of adaptability, but not full adaptability, may be more effective but more difficult to compute. Finally, the time duration of a trajectory is a measure of robustness to unknown disturbances as discussed in Section II.D. Therefore, constraints that make the aircraft arrive at its destination later are less robust.

The threshold set on the metric to decide if the flight is excessively constrained may be to some extent subjective, reflecting the decision maker's tolerance to the risk of constraint violation. The threshold may depend on the type of constraints and the criticality of meeting them. Thus different constraint situations may require different thresholds. The threshold also depends on the flexibility needed for the aircraft to meet other objectives, such as ones dictated by company and on board concerns. Therefore, determining values for such a threshold requires research and experimentation to investigate the acceptable risk under different conditions.

\section{Conclusions}

This paper presented a summary of research that has been conducted to investigate two newly proposed functions for the ATM system: trajectory flexibility preservation and trajectory constraint minimization. Trajectory flexibility preservation enables planning an aircraft's trajectory such that it preserves a requisite level of maneuvering flexibility in accommodating disturbances, caused for example by other traffic or by weather activity. Trajectory constraint minimization enables ground-based agents, in collaboration with air-based agents, to impose minimal constraints on trajectories to achieve ATM objectives, such as separation assurance and flow management. The concept hypothesizes that, by preserving trajectory flexibility, aggregate system objectives such as maintaining acceptable traffic complexity are naturally achieved, and that minimizing the constraints imposed on a trajectory, without jeopardizing the intended ATM objectives, increases its flexibility. The two functions offer a trajectoryoriented approach to managing traffic complexity, by explicitly planning aircraft trajectories such that their contribution to complexity is minimized.

The paper presented metrics for measuring trajectory flexibility in terms of robustness and adaptability to the risk of violating separation, airspace hazards, and traffic flow management constraints. This paper summarized previous results and presented new extensions to the trajectory flexibility metrics and their application in traffic complexity mitigation and constraint minimization. Using simple hypothetical scenarios, it was shown that using the trajectory flexibility metrics mitigated traffic complexity by inducing self organization in the traffic situation as well as reducing indicators of traffic complexity such as traffic entropy and dynamic density. It was also shown that the application of trajectory flexibility metrics resulted in distributing traffic over available altitudes and reducing the creation of secondary conflicts by bringing about implicit coordination between aircraft. Finally, it was demonstrated how the trajectory flexibility metrics can also be used to assess the amount of maneuverability afforded to the aircraft by the constraints imposed on it and hence its exposure to the risk of constraint violation. These metrics can be used to aid negotiating constraint relaxation by the flight crew and minimizing the amount of constraints imposed on the aircraft by the ground based traffic management system.

\section{References}

${ }^{1}$ Joint Planning and Development Office, "Next Generation Air Transportation System Integrated Plan," URL: http://www.jpdo.gov/library/NGATS_v1_1204r.pdf.

${ }^{2}$ Wing, D., "A Potentially Useful Role for Airborne Separation in 4D-Trajectory ATM Operations," Proceedings of the $5^{\text {th }}$ AIAA Aviation Technology Integration and Operations (ATIO) Conference, AIAA-2005-7336, 2005.

${ }^{3}$ Green, S. M., Bilimoria, K. D., and Ballin, M. G., "Distributed Air/Ground Traffic Management for En Route Flight Operations." Air Traffic Control Quarterly, Vol. 9, No. 4, 2001, pp. 259-285.

${ }^{4}$ Idris, H., Vivona, R., Penny, S., Krozel, J., and Bilimoria, K., "Operational Concept for Collaborative Traffic Flow Management based on Field Observations," Proceedings of the $5^{\text {th }}$ AIAA 5th Aviation Technology, Integration and Operations (ATIO) Conference, AIAA-2005-7434, 2005.

${ }^{5}$ Idris H., D. Wing, R. Vivona, and J.L. Garcia-Chico, "A Distributed Trajectory-Oriented Approach to Managing Traffic Complexity," Proceedings of the $7^{\text {th }}$ AIAA Aviation Technology Integration and Operations (ATIO) Conference, AIAA-2007-7731, 2007.

${ }^{6}$ Idris H., R. Vivona, J.L. Garcia-Chico, and D. Wing, "Distributed Traffic Complexity Management by Preserving Trajectory Flexibility," Proceeding of the 26 ${ }^{\text {th }}$ Digital Avionics Systems Conference, 2007. 
${ }^{7}$ Idris H, T. El-Wakil, and D. Wing, "Trajectory Planning by Preserving Flexibility: Metrics and Analysis," Proceedings of the AIAA Guidance Navigation and Control (GNC) Conference, AIAA-2008-7406, 2008.

${ }^{8}$ Idris H., and R. Vivona, "Metrics for Traffic Complexity Management in Self-Separation Operations," Air Traffic Control Quarterly, Volume 17, Number 1, 2009.

${ }^{9}$ Idris H., D. Delahaye, and D. Wing, "Distributed Traffic Flexibility Preservation for Traffic Complexity Mitigation ," $8^{\text {th }}$ USA/Europe Air Traffic Management R\&D Seminar, 2009.

${ }^{10}$ Idris H, N. Shen, T. El-Wakil, and D. Wing, “Analysis of Trajectory Flexibility Preservation Impact on Traffic Complexity," Proceedings of the AIAA Guidance Navigation and Control (GNC) Conference, 2009.

${ }^{11}$ Idris H, N. Shen, and D. Wing, "Improving Separation Assurance Stability through Trajectory Flexibility Preservation," Proceedings of the AIAA AIAA $10^{\text {th }}$ Aviation Technology, Integration and Operations (ATIO) Conference, AIAA-2010-????, 2010.

${ }^{12}$ Laudeman, I.V., Shelden, S.G., Branstrom, R., \& Brasil, C.L., 1999, Dynamic Density: An Air Traffic Management Metric, NASA-TM-1998-112226

${ }^{13}$ Sridhar, B., Sheth, K.S., \& Grabbe, S., Airspace Complexity and its Application in Air Traffic Management, $2^{\text {nd }}$ USA/Europe Air Traffic Management R\&D Seminar, Orlando, Florida

${ }^{14}$ Kopardekar. P. and S. Magyarits, "Measurements and prediction of dynamic density," $5^{\text {th }}$ USA/Europe ATM $R \& D$ Seminar, 2003.

${ }^{15}$ Davison, J., Histon, J., Ragnarsdottir, M., Major, L., Hansman, R.J., "Impact of Operating Context on the Use of Structure in Air Traffic Controller Cognitive Processes". $5^{\text {th }}$ USA/Europe Air Traffic Management R\&D Seminar, 2002.

${ }^{16}$ Delahaye, D. Puechmorel, S., Hansman, R.J., and Histon, J., "Air traffic complexity based on non linear dynamical systems", $5^{\text {th }}$ USA/Europe Air Traffic Management R\&D Seminar, 2003. 